\section{Simple Linear Regression Model Is Misleading When Used to Analyze Quantitative Diffusion Tensor Imaging Data That Include Young and Old Adults}

I read with interest a recent article published in this journal by Wang et al. ${ }^{1}$ The authors analyzed diffusion tensor imaging (DTI) data acquired on 71 healthy young, old, and older adult brains a (20-79 years of age). The authors calculated diffusion tensor metrics such as fractional anisotropy (FA) and mean, axial, and radial diffusivities by placing regions of interest on the caudate, putamen, and globus pallidus, and they used a linear regression model to fit the scatter of age versus DTI metrics. The article highlights the importance of using the tensor eigenvalues in the interpretation of normal-aging brain data in key gray matter structures that can be used as surrogate neuroimaging markers of natural aging. On the basis of the analysis of these regions of interest, the study concluded that FA increased steadily with age in the putamen $(r=0.535, P<.001)$. The FA increase in the putamen was attributed primarily to a decrease in the transverse diffusivity $(r=$ $-0.451, P<.008)$.

The increase in striatal FA with age as reported by Wang et al is an important finding that confirms previous and recent DTI reports on both healthy children ${ }^{2,3}$ and young ${ }^{3,4}$ and older adults, ${ }^{5-9}$ or across the human lifespan. ${ }^{10}$

While a trend in striatal increase in FA versus age reported by Wang et al is consistent with several reports using different DTI analysis methods, ${ }^{2-11}$ I should also point out that the finding of reduced mean diffusivity with age is contradictory to several previous reports that compared healthy young and older adults. For example, Bhagat and Beaulieu ${ }^{6}$ and Pfefferbaum et $\mathrm{al}^{7}$ reported that putaminal tensor axial and mean diffusivities increased significantly with advancing age. Càmara et $\mathrm{al}^{8}$ reported an increase in putaminal diffusion anisotropy but a nonsignificant trend in age versus mean diffusivity.

The expected rise in the water-molecular-diffusivity trend in deep striatal gray matter can be seen when including young children and adopting nonlinear curve-fitting models. ${ }^{10}$ The striatal mean diffusivity curves across the lifespan should also mimic the transverse relaxation age trajectories. ${ }^{11-13}$ The nonlinear (eg, quadratic) model consolidates reports on healthy children and young and older adults.

I conclude that DTI quantitative reports with a relatively small population and sparse attenuation and extended age ranges should not use simple linear regression because this simple model fails to accommodate the expected decrease in diffusivity in children and the predicted rise in diffusivity as a result of increased water extracellular mobility as tissue ages. ${ }^{11-13}$

\section{References}

1. Wang $\mathrm{Q}, \mathrm{Xu} \mathrm{X}$, Zhang $\mathrm{M}$. Normal aging in the basal ganglia evaluated by eigenvalues of diffusion tensor imaging. AJNR Am J Neuroradiol 2010;31: 516-20

2. Mukherjee P, Miller JH, Shimony JS, et al. Diffusion-tensor MR imaging of gray and white matter development during normal human brain maturation. AJNR Am J Neuroradiol 2002;23:1445-56

3. Snook L, Paulson LA, Roy D, et al. Diffusion tensor imaging of neurodevelopment in children and young adults. Neuroimage $2005 ; 26: 1164-73$

4. Lebel C, Walker L, Leemans A, et al Microstructural maturation of the human brain from childhood to adulthood. Neuroimage 2008;40:1044-55

5. Abe $\mathrm{O}$, Aoki S, Hayashi $\mathrm{N}$, et al. Normal aging in the central nervous system: quantitative MR diffusion tensor analysis. Neurobiol Aging 2002;23:433-41

6. Bhagat YA, Beaulieu C. Diffusion anisotropy in subcortical white matter and cortical gray matter: changes with aging and the role of CSF-suppression. J Magn Reson Imaging 2004;20:216-27

7. Pfefferbaum A, Adalsteinsson E, Rohlfing T, et al. Diffusion tensor imaging of deep gray matter brain structures: effects of age and iron concentration. Neurobiol Aging 2010;31:482-93

8. Càmara E, Bodammer N, Rodríguez-Fornells A, et al. Age-related water diffusion changes in human brain: a voxel-based approach. Neuroimage 2007;34: $1588-99$

9. Hasan KM, Halphen C, Boska MD, et al. Diffusion tensor metrics, T2 relaxation, and volumetry of the naturally aging human caudate nuclei in healthy young and middle-aged adults: possible implications for the neurobiology of human brain aging and disease. Magn Reson Med 2008;59:7-13

10. Hasan KM, Frye RE. Diffusion tensor-based regional gray matter tissue segmentation using the international consortium for brain mapping atlases. $\mathrm{Hu}$ man Brain Mapping. 2010 (in press)

11. Hasan KM, Walimuni IS, Abid H, et al. DTI, T2 relaxation and volumetry of the human brain corpus striatum across the lifespan. In: Proceedings of the 18th Meeting of the International Society for Magnetic Resonance in Medicine, Stockholm, Sweden. May 1-7, 2010: 606

12. Saito N, Sakai O, Ozonoff A, et al. Relaxo-volumetric multispectral quantitative magnetic resonance imaging of the brain over the human lifespan: global and regional aging patterns. Magn Reson Imaging 2009;27:895-906. Epub 2009 Jun 10

13. Baratti C, Barnett AS, Pierpaoli C. Comparative MR imaging study of brain maturation in kittens with $\mathrm{T} 1, \mathrm{~T} 2$, and the trace of the diffusion tensor. Radiology 1999;210:133-42

K.M. Hasan

Associate Professor of Diagnostic and Interventional Imaging Department of Diagnostic and Interventional Imaging University of Texas Medical School at Houston

Houston, Texas

DOI 10.3174/ajnr.A2184 